\title{
Good news for sound science-based alternatives in liver
}

\author{
Bernward Garthoff
}

Published online: 15 May 2011

(C) Springer-Verlag 2011

These days, several activities in the alternative methods research area seem to be much more target oriented than in the past decade when propagators of in vitro methodologies pushed for "alternative methods" to laboratory animal testing, not scientifically solid. And ironically, by that, they rather induced counter reactions than achieving the dissemination of valid alternatives (Garthoff 2005), but as usually, help arrives from other fields of science, in this case of transplantation medicine. Some of the work on alternative methodology reported by the group at the VUB Brussels in this issue is based on hard, long-time research work and only being made possible by novel techniques, thus giving also a basis for the application of stem cell technology in the future. It was a long-standing request for a perfused liver model, almost fully functionable, and thereby, serving the identification of potentially liver-damaging agents and pharmaceuticals at an early stage of development. Since we are close to reaching that goal, the article on methodology by de Kock et al. 2011 cannot be valued high enough in embarking on a novel approach to hepatic function analysis. The method of decellularization of intact rat liver (Shupe et al. 2010; Bao et al. 2010) is providing an intact decellularized organ with an intact liver capsule and all applications looked for; furthermore, re-cellularization of the tissue engineered liver graft enables liver-specific functions such as albumin secretion, urea synthesis, and cytochrome P 450 expression at comparable levels of "in vivo liver" (Uygun et al. 2010). Besides of the obvious potential for the generation of a transplantable liver graft as thera-

B. Garthoff $(\bowtie)$

BIO.NRW, Cluster Biotechnology North-Rhine Westphalia, Merowingerplatz 1, 40225 Düsseldorf, Germany

e-mail: b.garthoff@bio.nrw.de peutic principle for liver disease, this technical approach holds potential for in vitro applications in toxicology, pharmacology and alternative methods versus in vivo testing in laboratory animals. It needed quite some perfecting to obtain a feasible tool: extensive bioengineering was needed addressing the preservation of the extracellular matrix components and intact vascular network, as well as the introduction of human fetal liver and endothelial cells (Baptista et al. 2011). The development of a bioartificial, threedimensional in vitro model of a naturally derived liver scaffold closely resembling the in vivo architecture, however, is the major step into a new area of in vitro liver methodology, which holds promises for all kinds of applications in toxicology and drug development processes in the future. After the debate on possibilities and limitations of in vitro toxicology tests and replacement of in vivo studies in the European Chemical Policy Legislation process, Bolt and Hengstler (2008) had concluded the following, taking issue with 2 outstanding articles of 2008 in this journal covering hepatotoxicity and alternative methodology: Continue the existing (in vivo) methods until reliable in vitro techniques are available. Isolated perfused organs have had a longstanding usage in alternative methodology as valid methods and saved a lot of lab animal lives (just think of the Langendorff heart preparation in pharmacology of Otto Langendorff of 1895 still being modified and used, see for reference: Döring 1996): here comes the next and this time finally, it is liver.

\section{References}

Bao J, Shi Y, Sun H, Yin X, Li L, Chen X, Bu H (2010) Construction of a portal implantable functional tissue engineered liver using perfusion-decellularized matrix and hepatocytes in rats. Cell Transplant (in press) 
Baptista PM, Siddiqui MM, Lozier G, Rodriguez SR, Atala A, Soker S (2011) The use of whole organ decellularization for the generation of a vascular liver organoid. Hepatology 53(2):604-617

Bolt HM, Hengstler JG (2008) Most cited articles in the Archives of Toxicology: the debate about possibilities and limitations of in vitro toxicity tests and replacement of in vivo studies. Arch Toxicol 82:881-883

De Kock J, Ceelen L, De Spiegelaere W, Casteleyn C, Claes P, Vanhaecke T, Rogiers V (2011) Simple and quick method for whole liver decellularization: a novel in vitro three-dimensional bio-engineering tool? Arch Toxicol (this issue)

Döring HJ (1996) The isolated perfused heart according to Langendorff-History and presence, modifications and applications. Eur Surg/Acta Chir Austriaca 28(6):326-333
Garthoff B (2005) Alternatives to animal experimentation: the regulatory background. Toxicol Appl Pharmacol 207(2):388-392

Shupe T, Williams M, Brown A, Wittenberg B, Petersen BE (2010) Method for decellularization of intact rat liver. Organogenesis 6(2):134-136

Uygun BE, Soto-Gutierrez A, Yagi H, Izamis ML, Guzzardi MA, Shulman C, Milwid J, Kobayashi N, Tilles A, Berthiaume F, Hertl M, Nahmias Y, Yarmush ML, Uygun K (2010) Organ reengineering through development of a transplantable recellularized liver graft using decellularized liver matrix. Nat Med 16(7):814-820 